\title{
Real-Time Path Loss And Node Selection For Cooperative Localization In Wireless Sensor Networks
}

\author{
Albert Bel ${ }^{1}$, José López Vicario ${ }^{2}$, Gonzalo Seco-Granados ${ }^{3}$ \\ Group of Signal Processing for Communication and Navigation (SPCOMNAV) \\ Telecommunications and Systems Engineering Department, Universitat Autonoma de Barcelona (UAB) \\ Edifici $Q$ - Campus de la UAB, 08193 Bellaterra (Barcelona), Spain \\ Phone: +34 9358138 43, Fax +34 935814031 \\ 1 albert.bel@uab.cat \\ 2 jose.vicario@uab.cat \\ 3 gonzalo.seco@uab.cat
}

\begin{abstract}
RSS-based localization is considered a lowcomplexity algorithm with respect to other range techniques such as TOA or AOA. The accuracy of RSS methods depends on the suitability of the propagation models used for the actual propagation conditions. In indoor environments, in particular, it is very difficult to obtain a good propagation model. For that reason, we present a cooperative location algorithm that dynamically estimates the path loss exponent by using RSS measurements. Since the energy consumption is a key point in sensor networks, we propose a node selection in order to avoid the use of all the nodes in the network for positioning purposes. Hence, we derive a practical solution tailored to the strict requirements of sensor networks in terms of complexity, size and cost. We present results based on both computer simulations and experimentation with the Crossbow IRIS motes showing that the proposed scheme offers a good performance in terms of position accuracy.
\end{abstract}

\section{INTRODUCTION}

In recent years, location estimation in wireless sensor networks (WSN) has raised a lot of interest from researchers [1], [2], [3]. In that sense, cooperative location algorithms has meant an improvement in terms of estimation accuracy. Cooperative methods allow for the possibility of obtaining information metrics from all nodes of the network. This characteristic is quite useful because cooperative techniques can increase localization performance in terms of both accuracy and coverage, while keeping the terminals with reduced complexity.

Normally, the first phase of a location algorithm consists in the measurement of internode distances. Distance estimates are usually based on time of arrival (TOA) or received signal strength (RSS) metrics. Once all internode distances are estimated, the location algorithm starts a procedure in which

The work has been supported by the Spanish Government under project TEC2008-06305 and CENIT-SINTONIA, the Catalan Government under gran 2009 SGR 298, and the Chair of Knowledge and Technology Transfer Parc de Recerca UAB - Santander. each node only modify their own state through those estimated metrics and the nodes state information.

RSS-based distance estimations, in particular, are based on the well-known radio propagation path loss model. This technique has become the most inexpensive and simple one because RSS of RF signals can be measured during normal transmissions between nodes. However, distance estimates are degraded when the cooperation between distant nodes is allowed [4]. This is because, the error introduced in the measurements can be multiplicative to the distance [5]. Cooperation with a high number of nodes can affect the algorithm behavior when the distance between the nodes increases. Also, communication with more nodes will also increase the consumption of energy. In order to minimize these two effects the authors proposed in [6] a node selection strategy. More specifically, the presented scheme offered a good trade-off in terms of energy consumption vs. positioning accuracy.

Other sources of error affecting distance estimations based on RSS are shadowing and multipath signals, which complicate the modeling of the channel that nodes need to know a priori. In order to avoid the dependency on an estimated model obtained with previous off-line measurements, the introduction of real-time estimation of the propagation model is proposed in [7]. In particular, the authors derived a non-cooperative positioning algorithm which dynamically estimates the propagation models that best fit the propagation environments, by means of RSS measurements only.

In this paper, we focus on cooperative distributed localization method based on RSS. We propose the use of a realtime path loss determination. This allows us to eliminate the necessity of a calibration stage and the adaptation of the algorithm to possible changes in the environment not contemplated in previous measurement campaigns. Furthermore, two node selection criteria are proposed in order to reduce the number of cooperative nodes. By doing so, the scheme only selects those nodes providing accurate distance measurements. As a result, 


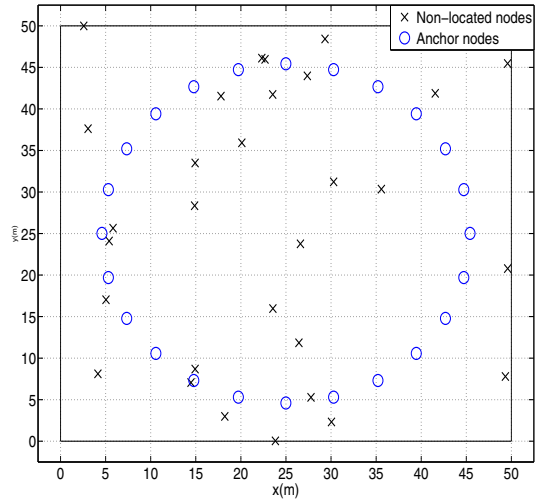

Fig. 1. Example of an scenario deployment

accuracy vs. energy consumption trade-off is significantly improved. As shown in the paper, experimental results carried out with Crossbow Iris motes validates the proposed scheme.

\section{System Model}

Let us consider a wireless sensor network with $N$ nodes. There are $N_{1}$ nodes, whose exact locations are known (anchor nodes). The rest of the nodes $N_{2}=N-N_{1}$ do not know their position (non-located nodes). The main goal is to estimate the location of the non-located nodes with the help of anchor nodes and the rest of nodes in the network by means of a cooperative strategy. Concerning the anchor nodes placement, we follow the approach presented in [8], where it is shown that the best anchor placement is a centered circumference of radius equal to the root-mean-square (RMS) of the nonlocated nodes distances to the center. One can see in Figure 1 an example of the scenario deployment.

\section{A. Measurement phase}

As previously commented, we consider an RSS-based distributed cooperative algorithm for location estimation. The first phase of the algorithm consists in obtaining internode distances through RSS measurements. Power received is modeled through the well-known radio-propagation path loss and shadowing model [4]. Received Signal Strength (RSS) can be expressed as the power received in node $j$ from a signal transmitted by node $i, P_{i j}$, as:

$$
R S S_{i j}=P_{i j}=P_{0}-10 \alpha \log _{10} d_{i j}-v_{i j}(d B m)
$$

where $P_{0}$ is the power received in $\mathrm{dBm}$ at $1 \mathrm{~m}$ distance, $d_{i j}$ is the distance between nodes $i$ and $j$, parameter $\alpha$ is the path loss exponent and $v_{i j} \sim \mathcal{N}\left(0, \sigma_{v}^{2}\right)$ represents lognormal shadow-fading effects, where the value of the standard deviation $\sigma_{v}$ depends on the characteristics of the environment. Given the power received $R S S_{i j}$ in (1), an estimate of the actual distance is derived as:

$$
\delta_{i j}=10^{\frac{P_{0}-R S S_{i j}}{10 \alpha}}
$$

\section{B. LS Cooperative algorithm}

Once the relative distances between nodes have been obtained, the position estimates for each non-located node are obtained by means of the Least-Squares criterion. Position estimates are calculated by obtaining the set of non-located node positions that minimize the Euclidean distance between estimated distances at the first phase and the distances computed using such position estimates. This is reflected in the following expression:

$$
C_{L S}(\mathbf{x})=\sum_{i=1}^{N_{2}} \sum_{j \in S_{i}}\left\|\delta_{i j}-\hat{d}_{i j}\right\|^{2}
$$

where $\hat{d}_{i j}=\left\|\hat{\mathbf{x}}_{\mathbf{i}}-\hat{\mathbf{x}}_{\mathbf{j}}\right\|$ is the distance between nodes $i$ and $j$, calculated with the estimated position (or real coordinates if node $j$ is an anchor) of nodes $i$ and $j, S_{i}$ is the number of nodes (anchor and non-located) that cooperates in the position estimation of non-located node $i$, and $\mathrm{x}$ are the coordinates of nodes.

The minimization of the cost function of (3) will be carried out with a distributed iterative method. We adopt a distributed strategy due to its scalability and robustness. Each nonlocated node iteratively computes, at each time instant $t$, its own coordinates $\hat{\mathbf{x}}_{i}$ by minimizing $C_{L S}$ through a distributed gradient descent mechanism:

$$
\hat{\mathbf{x}}_{i}(t)=\hat{\mathbf{x}}_{i}(t-1)+\gamma \sum_{j \in S_{i}}\left(\delta_{i j}-\hat{d}_{i j}\right) \mathbf{e}_{\mathbf{i j}}
$$

where $\mathbf{e}_{\mathbf{i j}}=\frac{\hat{\mathbf{x}}_{\mathbf{i}}-\hat{\mathbf{x}}_{\mathbf{j}}}{\left\|\hat{\mathbf{x}}_{\mathbf{i}}-\hat{\mathbf{x}}_{\mathbf{j}}\right\|}$ is the unit vector that takes the orientation between the node $i$ and node $j$ and $\gamma$ is the step size.

Several methods can be found in the literature to obtain appropriate initial values. In that direction, MultiDimensional Scaling (MDS) is proposed in [4] to obtain such values. It is a good option but it increases the computational cost. Another option can be based on a random initialization. It is a simple method but a higher number of iterations is required for algorithm convergence. We propose to initialize each $\hat{\mathbf{x}}_{\mathbf{i}}(0)$ as the coordinates of the nearest anchor node. When the node $i$ sets its own group $S_{i}$, it has to find the nearest anchor node and takes its coordinates as its own initial coordinate vector. This selection is done by selecting the anchor with the highest RSS. In the case that an anchor node is not present, random coordinates are selected. By doing so, an initial value closer to the final solution can be obtained without increasing the computational cost.

\section{Energy consumption model}

In this paper we also care of the energy consumption of the network. To do so, we consider the model of the total amount of energy consumed by the network pesented in [9]:

$$
\epsilon=\left(\mu_{R_{x}}+\mu_{T_{x}}\right)\left(\sum_{i=1}^{N_{2}} S_{i}-N_{2}\right) \kappa
$$

where $\kappa$ is the number of iterations of the algorithm and $\mu_{T_{x}}$ and $\mu_{R_{x}}$ are the energy consumption dedicated for peer to peer transmission and reception procedures, respectively. 


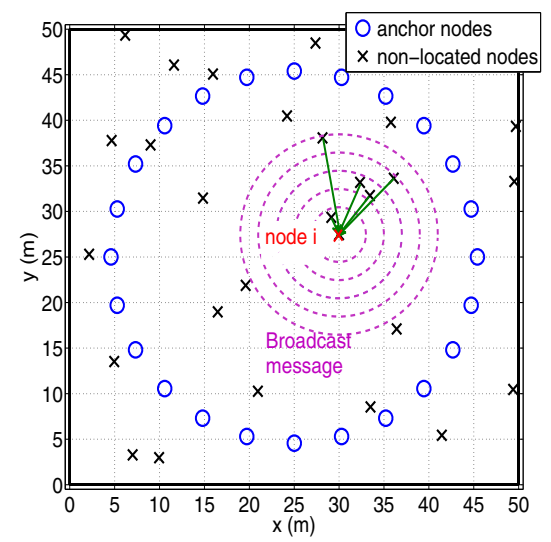

Fig. 2. Example of the creation of a group $S_{i}$

In order to create the cooperating group $S_{i}$ (see Figure 2), each node $i$, sends, at the beginning, a broadcast message with its coordinates $\mathbf{x}_{\mathbf{i}}$. Only those nodes that receive this message, answer with their node id and their location coordinates. With the received messages, each non-located node can create its own $S_{i}$ group. Once these groups are created, the exchange of messages are only done between cooperating nodes, as the energy consumption model above reflects. It is worth noting that energy consumption is an increasing function on the number of cooperating nodes $\left(S_{i}\right)$. For that reason, we consider a node selection algorithm aimed at selecting only the nodes with good channel conditions.

\section{Node Selection}

The authors presented in [6] a Node-selection Least Squares (NS-LS) location algorithm. The idea was to obtain a good trade-off in position accuracy versus energy consumption. As discussed in [6], the derivation of the optimal selection criterion is not possible. For that reason, the authors presented a sub-optimal scheme based on the received power threshold $\left(R S S_{t h}\right)$. In other words, only nodes with RSS higher than the $R S S_{t h}$ were allowed for cooperation. This criterion becomes in a simple scheme suitable for a hardware restricted WSN. In particular, the choice of the $R S S_{t h}$ value was designed to assure a minimum number of anchor nodes inside the cooperating nodes group $\left(S_{i}\right)$. In accordance to this value, $N_{m}$, different trade-off points in the energy consumption versus accuracy can be achieved. Results showed that $N_{m}$ equal to 3 allows the algorithm to achieve an excellent trade-off.

\section{Node SELECTION BASED ON REAL-TIME PATH LOSS ESTIMATION}

As commented above, the objective of this paper is the improvement of RSS-based localization algorithm. In particular, we combine node selection with real-time path loss estimation.

As shown in [6], node selection provides significant gain in terms of positioning accuracy and energy efficiency. Path loss estimation is introduced to further improve system performance.

\section{A. Motivation of Path Loss Estimation}

Consider a cooperative scenario where nodes use an RSSbased location algorithm. The use of such range metrics becomes in a simpler positioning procedure (in comparison with TOA). However, the use of RSS measurements requires an accurate scenario modeling. In order to obtain a good model, a thorough previous measurement campaign is needed. It is also necessary to consider all the environments where the algorithm can be used. By adopting a real-time estimation of the path loss exponent, the algorithm can be adapted to the scenario without the need of performing another measurement campaign. Thanks to real-time estimation we can also avoid the equal path loss exponent assumption for all links.

\section{B. Path Loss Exponent Estimation Through Real-Time RSS Measurements}

The main idea is to obtain an estimate of the path loss exponent through proper RSS measurements done in normal transmissions. It is important to remark that the use of RSS metrics do not introduce extra hardware requirements. With this idea in mind we could achieve a location algorithm able to adapt to the scenario variations.

The path loss estimates are obtained through the minimization of the following cost function [7]:

$$
C_{L S}\left(\left(\hat{\alpha}_{i 1}, \ldots, \hat{\alpha}_{i N_{S_{i}}}\right)\right)=\sum_{j \in S_{i}}\left\|10^{\frac{P_{0}-P_{i j}}{10 \alpha_{i j}}}-\hat{d}_{i j}\right\|^{2}
$$

The idea is to find the values of $\alpha_{i j}$ that minimizes the euclidean distance between estimate distances $\left(\delta_{i j}\right)$ and calculated distances $\left(\hat{d}_{i j}\right)$. In order to do that, it is necessary an initial estimate of the node coordinates. Once the initial estimate is calculated, we minimize de $C_{L S}$ of (6) for each node $i$. With the obtained path loss exponent estimations we can update distance estimates $\delta_{i j}=10^{\frac{P_{0}-P_{i j}}{10 \alpha_{i j}}}$ and recalculate coordinates estimation $\hat{\mathbf{x}}_{i}$. All these procedures will be repeated along time. A scheme of the procedure of the location algorithm can be found in the Algorithm 1. It is worth noting that the minimization problem in (7) is iteratively solved with a Levenberg-Marquardt method [7] in a distributed fashion. Both procedures are iterated until convergence, being $t_{i t e r} 1$ and $t_{\text {iter } 2}$ the required number of iterations to attain it.

\section{Selection Mechanisms}

In this subsection, we derive two node selection algorithm for the proposed cooperative localization scheme. Since in this work we consider the adoption of real-time path loss estimation, we need to adapt the scheme presented in [6] to the new context. The use of RSS thresholds, in particular, it is not the optimal choice in an environment where path loss exponents of the different links can be quite different: the node with the highest RSS is not necessary the better one.

In particular we present two node selection mechanisms that will depend on the path loss estimates obtained through the algorithm 1. With those selection mechanisms we try to reduce the number of nodes that will cooperate in the 


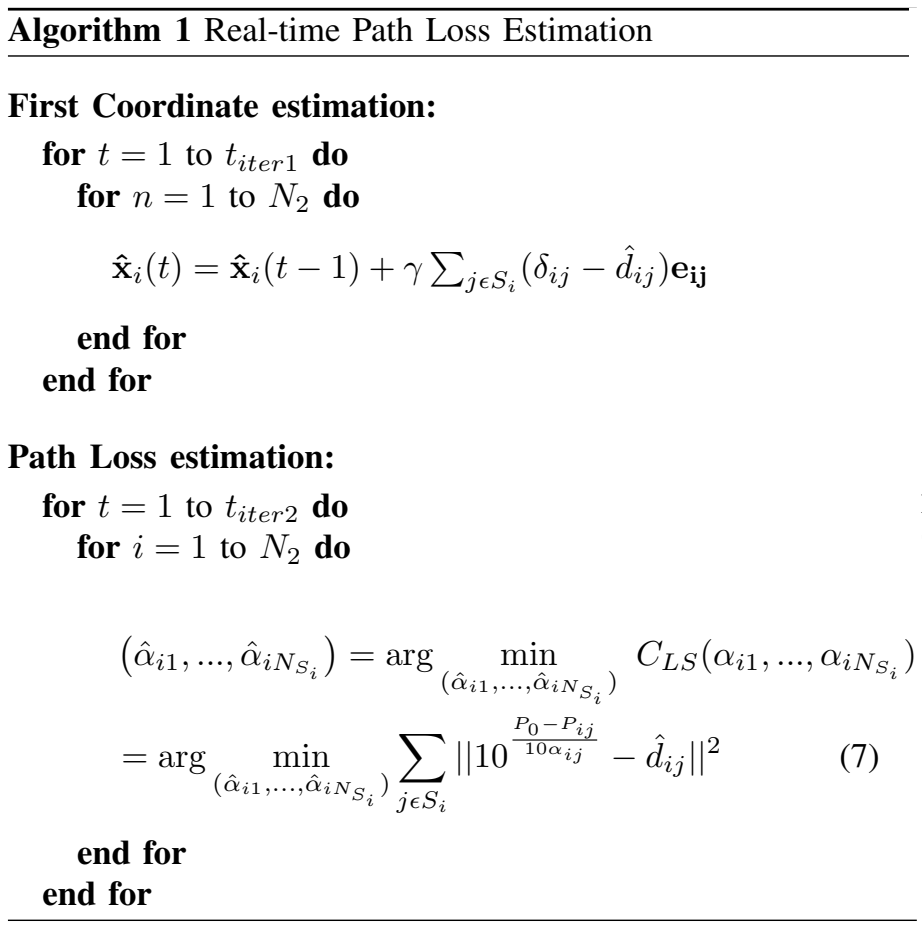

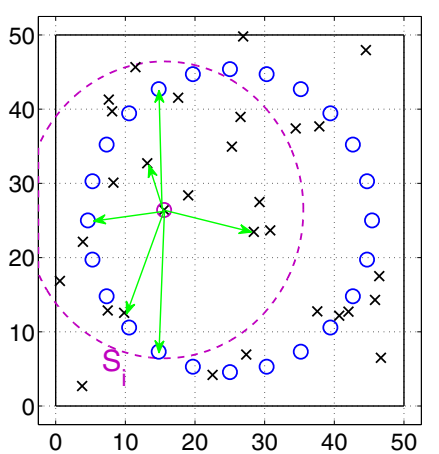

(a) Lower $\alpha$ selection

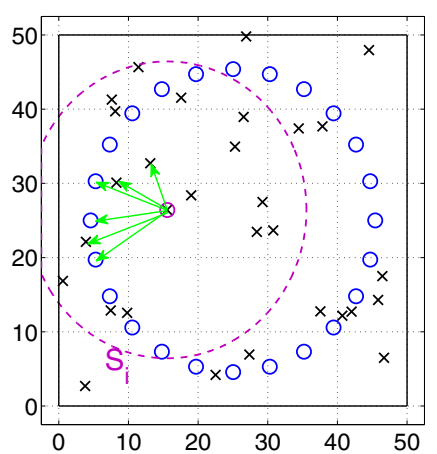

(b) Lower $\delta$ selection
Fig. 3. Example of both node selection methods

location algorithm presented in section II-B. The different criteria are: low-path loss selection and low-distance selection (see Figure 3). Hence, both node selection schemes depend on path loss estimates. On the one hand, with the low path loss selection we try to find those nodes with better channel conditions. On the other hand, the low distance criterion selects those nodes that have a low distance estimate $\delta_{i j}$. One can see in Figure 3(b) that these selection criterion could not select a nearer node, because distance estimates could not reflect the reality ( $\delta$ values depend on the quality of $\hat{\alpha}$ estimates).

- Low-path loss selection Given all the estimates of $\hat{\alpha}_{i j}$, the first selection mechanism selects those nodes that have the lowest values for the path loss exponent. In other words, by sorting the path loss exponents of the nodes

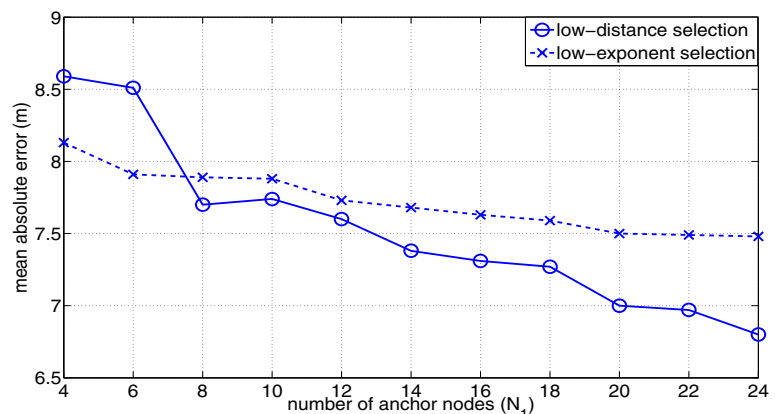

Fig. 4. Mean absolute error versus number of anchor nodes $\left(N_{1}\right)$ $\left(n_{\delta}=n_{\alpha}=6\right)$

inside the coverage of node $i$ :

$$
\hat{\alpha}_{i 1} \leq \hat{\alpha}_{i 2} \leq \ldots \leq \hat{\alpha}_{i n}
$$

being $\hat{\alpha}_{i 1}$ and $\hat{\alpha}_{i n}$ the lowest and highest exponent, respectively. We select the nodes with the lowest values:

$$
S_{i}^{N S}=\left(i 1, i 2, \ldots, i n_{\alpha}\right)
$$

with $n_{\alpha}$ standing for the number of selected nodes. With this selection mechanism we are interested in the selection of nodes that have better propagation conditions.

- Low-distance selection Now, the idea is to select the closest nodes to node $i$. We want to use nearer nodes in order to reduce distance error estimates, because, as shown in [5], the shadowing effect introduces errors multiplicative to the distance. By sorting now the distance estimate of the nodes inside the coverage of node $i$ :

$$
\delta_{i 1} \leq \delta_{i 2} \leq \ldots \leq \delta_{i n} \quad 1, \ldots, n \in S_{i}
$$

being, in this case, $\delta_{i 1}$ and $\delta_{i n}$ the lowest and highest distance estimate, respectively. The new group of cooperating nodes becomes:

$$
S_{i}^{N S}=\left(i 1, i 2, \ldots, i n_{\delta}\right)
$$

with $n_{\alpha}$ standing for the number of selected nodes.

In order to compare both mechanisms, in Figure 4 it is plotted the mean absolute error versus the number of anchor nodes $\left(N_{1}\right)$. One can see that the low-distance selection achieves a better performance for values of $N_{1}$ higher than 8 . Low-path loss selected nodes are not necessarily closer nodes to node $i$ (see Figure 3(a)). The distance error estimate will be higher than those obtained with a nearer node, although its propagation conditions are better (see Figure 3(b)). For that reason, it is observed that is better to use lower distance selection in order to achieve a higher performance gains. Given the better results obtained with the second mechanism, we will focus on the low-distance selection strategy in the sequel. The next step is to determine what is the optimal choice of $n_{\delta}$. To do so, we plot in Figure 5 the probability of obtaining positioning errors higher than a given threshold, 


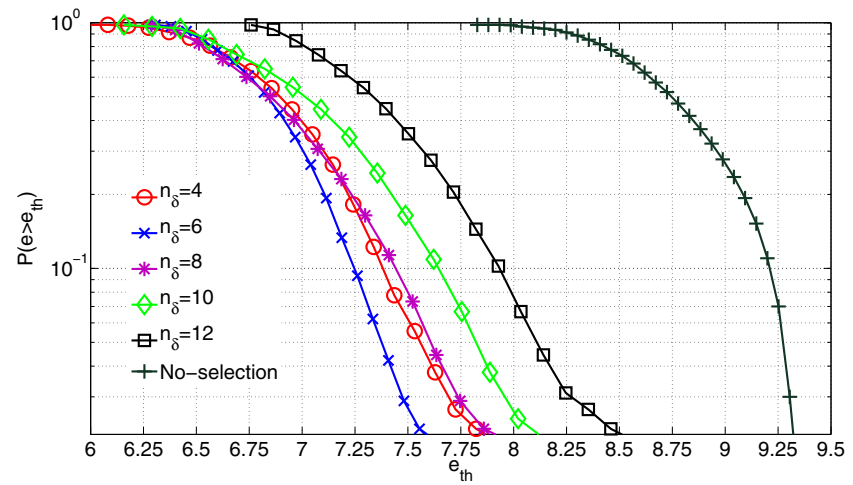

Fig. 5. Outage probability $\left(N_{1}=24\right)$

$e_{t h}$, for different values of $n_{\delta}$. As observed, better behavior is obtained with 6 nodes and, for that reason, we will consider this value in the next section.

Having a low value of cooperating nodes reduces the possibility of having anchor nodes (anchors are nodes with truly information of their exact location) inside the cooperating group. On the other hand, having more than 6 cooperating nodes implies the existence of further nodes. This fact implies a higher error of the distance estimates. It is important to achieve a good trade-off between having a group big enough to lodge an anchor but low enough not to use further nodes with a high error at distance estimates.

\section{NUMERICAL RESULTS}

This section presents the performance of the proposed node selection location algorithm with path loss estimation. We present, both simulations and experimental results obtained in an indoor scenario. The experimental and simulation parameters are shown in Table I.

\section{A. Computer simulation}

In this paper, we assume a more realistic scenario to that presented in [6] by allowing a different path loss exponent at each link (takes values between 2 and 5, see Table I). For that reason, we start the analysis by comparing our scheme with an algorithm that assumes equal path loss exponent for all the

TABLE I

SIMULATION PARAMETERS

\begin{tabular}{lc}
\hline \hline Simulation Parameters & Parameter Value \\
\hline \hline Size of sensor field & $50 \mathrm{~m} \mathrm{x} \mathrm{50} \mathrm{\textrm {m }}$ \\
Number of non-located nodes $\left(N_{2}\right)$ & 30 \\
Path Loss exponent $\alpha$ & $2-5$ \\
Standard deviation $\sigma_{v}$ & $4 \mathrm{~dB}$ \\
First-meter RSS $P_{0}$ & $-40 \mathrm{dBm}$ \\
Minimum $R S S$ (IRIS hardware constraint) & $-96 \mathrm{dBm}$ \\
Anchor radius & $20.4 \mathrm{~m}$ \\
energy consumption to transmit or receive $\mu_{T_{x}}$ or $\mu_{R_{x}}$ & $400 \mathrm{~nJ}$ \\
$n_{\delta}$ & 6 \\
\hline
\end{tabular}

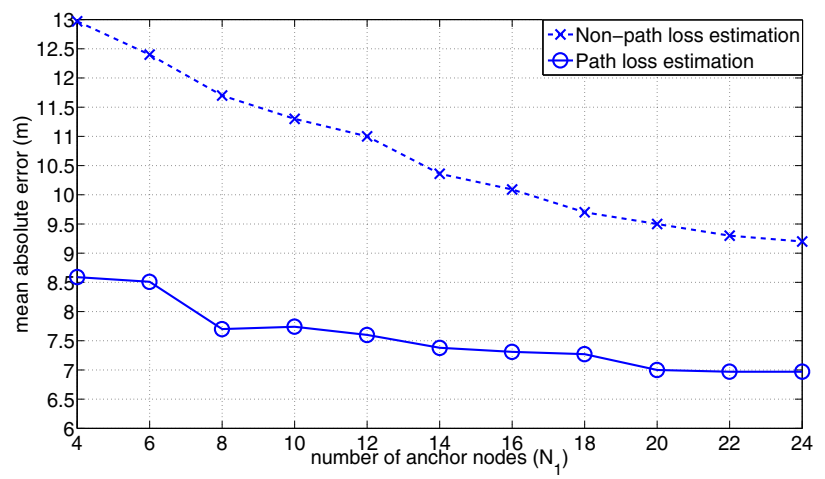

Fig. 6. Mean absolute error versus number of anchor nodes $\left(N_{1}\right)$ (solid line: lower distance selection, dashed line: non path loss estimation)

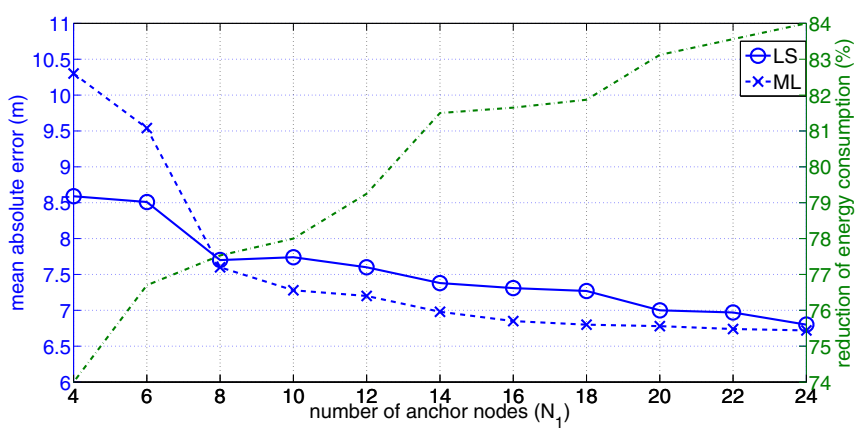

Fig. 7. Mean absolute error versus number of anchor nodes $\left(N_{1}\right)$ (solid line: Least Squares, dashed line: Maximum Likelihood, dashed-dotted line: energy reduction (\%))

links. In Figure 6, the mean absolute error versus the number of anchor nodes $\left(N_{1}\right)$ for both algorithms it is plotted. The different performance between our scheme and the algorithm without path loss estimation is remarkable. One can see that having different values of $\alpha$ for all links but assuming a constant value of $\alpha$ for distance estimation results in poorer performance, with respect to results obtained with path loss estimation. In particular, our scheme provides a $34.6 \%$ of gain with $N_{1}=4$ and $24.3 \%$ with $N_{1}=24$.

It is demonstrated that assuming an equal distance-power gradient for all links is not an appropriate choice.

Next, we compare the previous result (obtained with a Node Selection Least Squares algorithm) with those provided by a positioning system adopting real-time path loss estimation but based on Maximum Likelihood (ML) without node selection. In particular we compare both distributed methods in terms of position accuracy (see Figure 7).

A similar behavior is observed for both schemes in terms of positioning accuracy. For $N_{1}$ values below 8 our least square algorithm achieves better performance results. The importance of selecting only the nearer nodes is remarked when the 


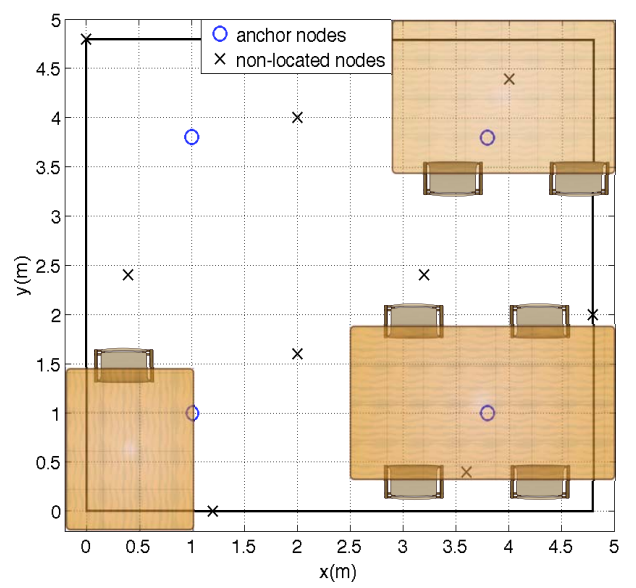

Fig. 8. Scenario deployed

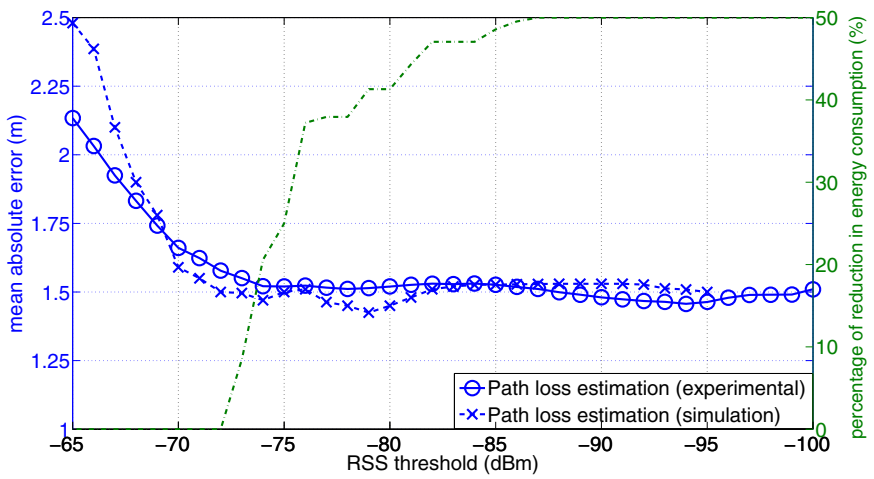

Fig. 9. Results of the mean absolute error and energy reduction vs. RSS threshold in a real indoor scenario (solid line: experimental results, dashed line: simulation results, dashed-dotted line: energy reduction (\%))

number of anchor nodes is low. On the other hand, for high values the ML location algorithm outperforms our scheme. However, similar results are observed for both schemes in terms of positioning accuracy.

In summary, our scheme based on LS and node selection provides similar results to that obtained with ML. Besides, our scheme is more efficient in terms of energy consumption as the number of cooperating nodes is limited (as observed in the figure we also show the energy consumption reduction with node selection).

\section{B. Experimental Validation}

In order to check the performance in a real scenario, we have carried out measurements in an indoor scenario with the IRIS motes@2.4GHz of Crossbow [10], where the total number of nodes with unknown position, $N_{2}$, is 9 (see Figure 8). The minimum $R S S_{\text {threshold }}(-96 \mathrm{dBm})$ is the sensitivity of the IRIS motes [10] used in the experimental results.
In Figure 9, it is plotted a comparison between the performance obtained with the measurements of the Iris motes and the performance obtained with a simulation of the same scenario. It is shown that simulations obtain the same performance in terms of positioning error. The error that we achieve is, approximately, 1.5 meters in both results. As observed, simulation results are quite similar to the experimental one. This fact validates the simulations carried out before.

Finally, it is worth noting that the values of the error obtained here are lower than the values obtained in previous computer simulations. This is justified by the fact that the experimental results are done for a small scale network.

\section{CONCLUSION}

In this paper we have proposed a cooperative RSS-based location algorithm with real-time path loss exponent estimation. The results achieved reveal the importance of having an accurate propagation model. Performance obtained with path loss estimation is significantly better than assuming a constant path loss exponent. Furthermore the necessity of an offline calibration is avoided with the presented solution. Since wireless sensor networks are energy-constrained networks, we have also presented a node selection criterion that reduces the number of cooperating nodes. It has been shown that having a reduced number of nodes cooperating allows us to reduce the energy consumption without affecting the accuracy. Besides, we have presented experimental results that validates the proposed method and we have compared it with ML showing the efficiency of the solution.

\section{REFERENCES}

[1] J. Bachrach and C. Taylor, Localization in Sensor Networks. Wiley and Sons, 2005.

[2] A. Boukerche, H. Oliveira, E. Nakamura, and A. Loureiro, "Localization systems for wireless sensor networks," Wireless Communications, IEEE, vol. 14, no. 6, pp. 6-12, December 2007.

[3] H. Wymeersch, J. Lien, and M. Win, "Cooperative localization in wireless networks," Proceedings of the IEEE, vol. 97, no. 2, pp. 427450, Feb. 2009.

[4] X. Li, "Collaborative localization with received-signal strength in wireless sensor networks," Vehicular Technology, IEEE Transactions on, vol. 56, no. 6, pp. 3807-3817, Nov. 2007.

[5] N. Patwari, J. Ash, S. Kyperountas, I. Hero, A.O., R. Moses, and N. Correal, "Locating the nodes: cooperative localization in wireless sensor networks," Signal Processing Magazine, IEEE, vol. 22, no. 4, pp. 54-69, July 2005.

[6] A. Bel, J. L. Vicario, and G. Seco-Granados, "Node selection for cooperative localization: Efficient energy vs. accuracy trade-off'," in accepted in ISWPC 2010, May 2010.

[7] S. Mazuelas, A. Bahillo, R. Lorenzo, P. Fernandez, F. Lago, E. Garcia, J. Blas, and E. Abril, "Robust indoor positioning provided by real-time rssi values in unmodified wlan networks," Selected Topics in Signal Processing, IEEE Journal of, vol. 3, no. 5, pp. 821 -831, oct. 2009.

[8] J. Ash and R. Moses, "On optimal anchor node placement in sensor localization by optimization of subspace principal angles," in Acoustics, Speech and Signal Processing, 2008. ICASSP 2008. IEEE International Conference on, 31 2008-April 4 2008, pp. 2289-2292.

[9] Y. Zou and K. Chakrabarty, "Energy-aware target localization in wireless sensor networks," in Pervasive Computing and Communications, 2003. (PerCom 2003). Proceedings of the First IEEE International Conference on, March 2003, pp. 60-67.

[10] Crossbow technology. [Online]. Available: http://www.xbow.com/ 